nephron

Practice
Nephron 2015;131:227-236

DOI: $10.1159 / 000441956$
Received: May 27, 2015

Accepted after revision: October 23, 2015

Published online: November 17, 2015

\title{
Categories of Hospital-Associated Acute Kidney Injury: Time Course of Changes in Serum Creatinine Values
}

\author{
David G. Warnock ${ }^{a}$ T. Clark Powell ${ }^{b}$ John P. Donnelly, d Henry E. Wang ${ }^{\text {e }}$ \\ a Department of Medicine, ${ }^{b}$ School of Medicine, 'Division of Preventive Medicine, ${ }^{d}$ Department of Epidemiology, \\ School of Public Health, and ${ }^{e}$ Department of Emergency Medicine, University of Alabama at Birmingham, \\ Birmingham, Ala., USA
}

\section{Key Words}

Serum creatinine - Multivariable regression models .

Epidemiology

\begin{abstract}
Hospital-associated acute kidney injury (HA-AKI) is associated with increased inpatient mortality. Our objective was to categorize HA-AKI based on the timing of minimum and peak inpatient serum creatinine $(\mathrm{s} C \mathrm{r})$ and describe the association with inpatient mortality. Materials and Methods: This study is a retrospective analysis of an administrative data set for adults admitted to a single medical center for over 4 years. Changes and timing of the minimum and peak sCr were used to define HA-AKI categories. Results: Peak creatinine followed minimum creatinine for HA-AKI, and preceded the minimum value for transient HA-AKI (THA-AKI). A subset of patients developed HA-AKI after recovering from THA-AKI. Multivariable Cox regression analyses examined the association between these categories and 28-day inpatient mortality, adjusting for age, sex, race, Charlson comorbidity index, baseline kidney function, AKI recovery and renal replacement therapy. There were 50,601 patients included in the analyses, and 29,996 (59\%) did not have AKI. There were 2,440 deaths; $\mathrm{HA}-\mathrm{AKI}$ had a 2.24 -fold $(95 \% \mathrm{Cl} 1.99-2.51)$ increased risk, while THA-AKI group $(12,101)$ had a 1.23 -fold
\end{abstract}

(95\% Cl 1.09-1.40) increased risk for inpatient mortality. THA-AKI patients who recovered and then developed HAAKI had the same mortality risk as THA-AKI (1.27-fold [ $95 \% \mathrm{CI}$ 1.07-1.51]) but longer hospitalization and less recovery from AKI. Conclusions: Risk of short-term inpatient mortality is associated with $\mathrm{AKI}$, and this risk is attenuated with recovery of kidney function in the hospital. Systematic surveillance with repeated inpatient $\mathrm{s} C r$ values is needed to assess the shortand long-term consequences of HA-AKI.

(C) 2015 S. Karger AG, Basel

\section{Introduction}

Acute kidney injury (AKI) has significant sequelae including increased inpatient mortality, and long-term risk of renal and cardiovascular events and death [1]. AKI is common among critically ill patients [2-5]. Once AKI was ascertained based on changes in serum creatinine ( $\mathrm{sCr}$ ) values rather than diagnostic-related groups, the striking incidence of AKI became apparent [6].

Contribution from the AKI \& CRRT 2015 Symposium at the 20th International Conference on Advances in Critical Care Nephrology, Manchester Grand Hyatt, San Diego, Calif., USA, February 17-20, 2015.

\section{KARGER 125}

(c) 2015 S. Karger AG, Base

$1660-8151 / 15 / 1314-0227 \$ 39.50 / 0$

E-Mail karger@karger.com

www.karger.com/nef
Prof. David G. Warnock

Department of Medicine, University of Alabama at Birmingham

Room 614 ZRB, 1720 2nd Avenue South

Birmingham, AL 35294-0007 (USA)

E-Mail dwarnock@uab.edu 
The definition of $\mathrm{AKI}$ is based on a change in $\mathrm{sCr}$ from baseline values or a surrogate value if baseline values are not available $[7,8]$. Hospital-associated AKI (HA-AKI) is characterized by maximal ('peak') sCr values compared to the baseline value $[3,9]$. 'Transient azotemia' has been used to describe elevated $\mathrm{sCr}$ that normalizes within 3 days of admission without renal replacement therapy (RRT) [10] in contrast to patients with HA-AKI who often have persistently elevated $\mathrm{sCr}$ at the time of discharge [10-12]. Transient HA-AKI (THA-AKI) has been described an independent predictor of hospital mortality (OR 2.3 compared to patients without AKI), while persisting AKI was associated with a 6.1-fold risk for inpatient mortality [10].

We retrospectively analyzed an administrative data set from a single large referral center (University of Alabama Hospital at Birmingham (UAHB)) to examine the classification of AKI and its association with inpatient mortality, using the minimum $\mathrm{sCr}$ during the admission as the surrogate for baseline sCr. We hypothesized that the timing of minimum $\mathrm{sCr}$ relative to the peak $\mathrm{sCr}$ could distinguish between THA-AKI and HA-AKI. Accordingly, our objectives were to (1) distinguish between AKI categories, using the temporal relation between minimum and peak sCr, and (2) describe the associations of the AKI categories with inpatient mortality.

\section{Materials and Methods}

We examined hospital discharges from UAHB, a 1,121-bed academic healthcare center between October 1, 2009, and September 30, 2013, using demographic information, admissions and discharge dates, admission site location code, hospital inpatient service location, discharge diagnoses and procedure codes and discharge vital status. Each admission was linked to UAHB's central laboratory, and $\mathrm{sCr}$ values were extracted with date-time stamps and service locations. The Institutional Review Board at UAHB approved the use of de-identified patient information for this study and waived the requirement for informed consent at the patient level.

There were 109,456 patients (age $\geq 18$ years) discharged from UAHB (181,574 admissions), with 5,452 inpatient deaths (3.0\%) during the ascertainment interval. Patients with chronic dialysis or kidney transplantation or those having less than $2 \mathrm{sCr}$ measurements were excluded from the analysis. Cases were excluded for $\mathrm{sCr}<0.3 \mathrm{mg} / \mathrm{dl}$, estimated glomerular filtration rates (eGFRs) $<10$ $\mathrm{ml} / \mathrm{min} / 1.73 \mathrm{~m}^{2}$ and length of stay (LOS) $<1$ day [4]. Four cases were excluded with apparent miscoding of inpatient RRT based on $<0.2 \mathrm{mg} / \mathrm{dl}$ difference between first $\mathrm{sCr}$, peak sCr, minimum $\mathrm{sCr}$ and last $\mathrm{sCr}$.

Admission point of origin was determined from the site location code included in the discharge summary [13]. There were 11,065 patients transferred to the UAHB from other hospitals and 939 from other healthcare facilities (HCFs; e.g., skilled nursing homes, hospice units, psychiatry unit, rehabilitation unit, etc.); these 12,004 patients are defined as admissions originating from HCFs. There were 38,576 patients admitted to the UAHB from community locations (including 20,931 from home or office, 12,821 from outpatient physician offices or clinics and 4,725 patients from UAHB Emergency Department). There were 122 patients with unknown points of origin who were excluded from analysis. The follow-up period was limited to 28-day maximum LOS. The final analytic data set included 50,580 patients who were admitted once during the evaluation period.

The admission date-time stamp was assigned to that of the first sCr and was adjusted by $24 \mathrm{~h}$ for each day that the first sCr measurement was obtained after the admission date. HA-AKI was defined as cases where the minimum $\mathrm{sCr}$ preceding the highest measured ('peak') sCr value for the admission. We used an increment of $0.3 \mathrm{mg} / \mathrm{dl}$ as the criteria for AKI based on prior studies of the minimum increment independently associated with risk of 28-day inpatient mortality $[3,9,14]$. THA-AKI was defined with the highest ('peak') sCr preceding the minimum sCr, with a subsequent $\mathrm{sCr}$ decrease of $\geq 0.3 \mathrm{mg} / \mathrm{dl}$ from the peak sCr. Some THA-AKI patients subsequently developed HA-AKI following recovery to the minimum sCr value and were then classified as $2 \mathrm{HA}-\mathrm{AKI}$. Patients without a clinically significant change $( \pm 0.3 \mathrm{mg} / \mathrm{dl})$ between peak and minimum sCr were classified as not having AKI (No-AKI).

For time-to-event regression analysis, AKI category was the effect variable, and the exposure period was LOS ( $>1$ and $\leq 28$ days). The covariates included age stratified at the median value of 55 years, gender, black or non-black race, Charlson comorbidity index [15] and eGFR (stratified at $60 \mathrm{ml} / \mathrm{min} / 1.73 \mathrm{~m}^{2}$, where the CKD-EPI eGFR [16] was calculated from the minimum inpatient $\mathrm{sCr}$, provision of RRT and covariates that described the extent of recovery from peak $\mathrm{sCr}$ ). The outcome measure was inpatient mortality, ascertained as vital status at time of discharge or at the 28 th day of stay. We calculated the hazard ratios (HRs) and 95\% confidence intervals for inpatient mortality among cases with no AKI (reference) and among AKI categories. We compared baseline characteristics of patients using chi-square test and analysis of variance. Dunnett tests were used for multiple comparisons, with minimal statistical significance set at $\mathrm{p} \leq 0.05$. Mean values are presented as \pm 1 standard deviation, and medians with 25 th and 75th centiles. Survivor functions for multivariable-adjusted Cox analysis with point-wise confidence intervals were generated as described [17]. Relative integrated discrimination improvement (IDI) was used with an a priori criterion for clinical significance of $10 \%[18,19]$. Statistical analyses were performed with Stata version 13.1 (Stata Corp, College Station, Tex., USA) using the following functions: group, IDIsurv, median test, pwmean, prtest, stcox (with tvc option) and survci.

\section{Results}

The patient characteristics, classified by AKI category, are shown in table 1: 29,989 (59\%) did not develop AKI (No-AKI); 12,101 (24\%) had THA-AKI; 2,243 (4.4\%) developed HA-AKI after recovering from THA-AKI (2HAAKI) and 6,247 (12\%) developed HA-AKI. Compared to the No-AKI reference group, patients with AKI were old- 
Table 1. Characteristics of 50,580 inpatients (2,500 deaths, 4.9\%) without or with AKI, including discharge diagnosis groups

\begin{tabular}{|c|c|c|c|c|}
\hline & \multicolumn{4}{|l|}{ AKI category } \\
\hline & $\begin{array}{l}\text { No-AKI: } \\
29,989 \text { (59) }\end{array}$ & $\begin{array}{l}\text { THA-AKI: } \\
12,101(24)\end{array}$ & $\begin{array}{l}\text { 2HA-AKI: } \\
2,243 \text { (4.4) }\end{array}$ & $\begin{array}{l}\text { HA-AKI: } \\
6,247 \text { (12) }\end{array}$ \\
\hline Age, years & $53 \pm 18$ & $55 \pm 18$ & $56 \pm 18^{\mathrm{a}}$ & $58 \pm 17^{\mathrm{a}}$ \\
\hline Male sex & $15,028(50)$ & $7,270(60)$ & $1,408(63)$ & $3,408(55)^{\mathrm{a}}$ \\
\hline Black race ${ }^{\mathrm{b}}$ & 8,673 (29) & $3,933(33)$ & $465(36)$ & $1,998(32)^{a}$ \\
\hline Baseline eGFR ${ }^{c}$ & $100 \pm 27$ & $94 \pm 35$ & $95 \pm 40$ & $87 \pm 34^{\mathrm{a}}$ \\
\hline HCF transfers & $6,336(21)$ & $2,941(24)$ & $722(32)$ & $2,005(32)^{\mathrm{a}}$ \\
\hline LOS, days & $3.7 \pm 3.2$ & $6.9 \pm 5.2$ & $12 \pm 6.5$ & $8.1 \pm 6.1$ \\
\hline Charlson score & $1.2 \pm 1.7$ & $1.6 \pm 1.9$ & $2.0 \pm 2.1$ & $2.1 \pm 2.1^{\mathrm{a}}$ \\
\hline Diabetes & $5,089(17)$ & $2,697(22)$ & $466(21)$ & $1,553(25)^{\mathrm{a}}$ \\
\hline COPD & $4,459(15)$ & $1.875(16)$ & $397(18)$ & $1,140(18)^{\mathrm{a}}$ \\
\hline $\mathrm{CHF}$ & $2,104(7.0)$ & $1,429(12)$ & $434(19)$ & $1,312(21)^{a}$ \\
\hline Stroke & $2,534(8.5)$ & $994(8.2)$ & $241(11)$ & $631(10)^{\mathrm{a}}$ \\
\hline Renal disease & $1,139(3.8)$ & $1,681(14)$ & $492(22)$ & $970(16)$ \\
\hline AMI & $1,893(6.3)$ & 1,057 (8.7) & $265(12)$ & $842(14)$ \\
\hline Cancer & $2,263(7.6)$ & $854(7.1)$ & $154(6.9)$ & $549(8.8)^{\mathrm{a}}$ \\
\hline Met cancer & $1,394(4.7)$ & $590(4.9)$ & $87(3.8)$ & $361(5.8)^{\mathrm{a}}$ \\
\hline Inpatient RRT & 0 & $287(2.4)$ & $246(11)$ & $256(4.1)$ \\
\hline Ever in ICU & $5,280(18)$ & $4,564(38)$ & $1,425(64)$ & $3,028(49)^{a}$ \\
\hline Inpatient deaths & $506(1.7)$ & $606(5.1)$ & $267(12)$ & $1,121(18)^{\mathrm{a}}$ \\
\hline
\end{tabular}

Unless otherwise indicated, values are given as number (percentage) or means \pm SD; medians are given with (25th-75th centile).

SIRR = Incident rate ratio (incident rates express as events per 28 patient-days).

$p<0.001$ for comparisons of characteristics between each group by ANOVA for means, signed-rank test for medians and chi-square test for proportions; shown as bold text, relative to 'No-AKI', which served as the reference group.

${ }^{a} \mathrm{p}<0.05$ for comparison between THA-AKI, 2HA-AKI and HA-AKI (Dunnett test for multiple comparisons) or chi-square test or proportions.

${ }^{b}$ Race or ethnic group was self-reported, and further classified as black or non-black.

${ }^{c}$ eGFR calculated with minimum sCr.

COPD = Chronic obstructive pulmonary disease CHF = congestive heart failure; $\mathrm{AMI}=$ acute myocardial infarction.

er, more likely to be male, black, had longer LOS, higher Charlson comorbidity scores, more likely to spend at least 1 day in an intensive care unit (ICU) and more likely to be transferred from another HCF to UAHB.

There were differences in the Charlson comorbidities between AKI subgroups, especially for diabetes, congestive heart failure and acute myocardial infarction. The prevalence of chronic renal disease was higher, which was reflected in lower baseline eGFR values for each patient. The inpatient mortality rates were $1.7 \%$ for NoAKI, $5.1 \%$ for THA-AKI, $12 \%$ for $2 \mathrm{HA}-\mathrm{AKI}$ and $18 \%$ for HA-AKI.

Figure 1 illustrates the time course of $\mathrm{sCr}$ changes for the AKI categories. HA-AKI, 2HA-AKI and THA-AKI were defined based on the timing of the peak and mini- mum sCr; the peak sCr preceded the minimum $\mathrm{sCr}$ for THA-AKI (blue) and 2HA-AKI (purple), while the minimum sCr preceded the peak $\mathrm{sCr}$ for HA-AKI (red). The inset shows elapsed time (hours) relative to the estimated time of admission.

For THA-AKI and 2HA-AKI, the peak sCr was recorded at $38 \pm 47$ and $30 \pm 36 \mathrm{~h}$ after admission, while the minimum sCr values were observed at for $115 \pm 88$ and $136 \pm 97 \mathrm{~h}$ after admission, respectively. The peak sCr for HA-AKI occurred at $127 \pm 105 \mathrm{~h}$, and followed the minimum sCr recorded at $42 \pm 59 \mathrm{~h}$. For all AKI categories, the discharge time was 7-10 $\mathrm{h}$ after the last recorded $\mathrm{sCr}$ (fig. linset).

The distinguishing characteristic of the 2HA-AKI patients was progression to a secondary peak $\mathrm{sCr}$ (peak 2) 
Fig. 1. Time course for AKI types. The analysis pertains to 50,601 patients with a single admission to the University of Alabama at Birmingham Hospital between October 1, 2009 and September 30, 2013; first, peak, minimum and last $\mathrm{sCr}$ values, connected by dashed lines, stratified by AKI type. \#1, first sCr recorded for admission; 2HA-AKI, transient hospital associated AKI that resolves, followed by a second episode of AKI; THA-AKI defined as peak $\mathrm{sCr} \geq 0.3 \mathrm{mg} / \mathrm{dl}$ above the minimum $s C r$ with peak sCr preceding the minimum sCr; HA-AKI defined as peak $\mathrm{sCr} \geq 0.3 \mathrm{mg} /$ $\mathrm{dl}$ above the minimum sCr with minimum $\mathrm{sCr}$ preceding the peak $\mathrm{sCr}$; last, last $\mathrm{sCr}$ recorded for admission; No-AKI defined as peak $\mathrm{sCr}<0.3 \mathrm{mg} / \mathrm{dl}$ above the minimum sCr. The elapsed hours relative to admission are listed in the inset.

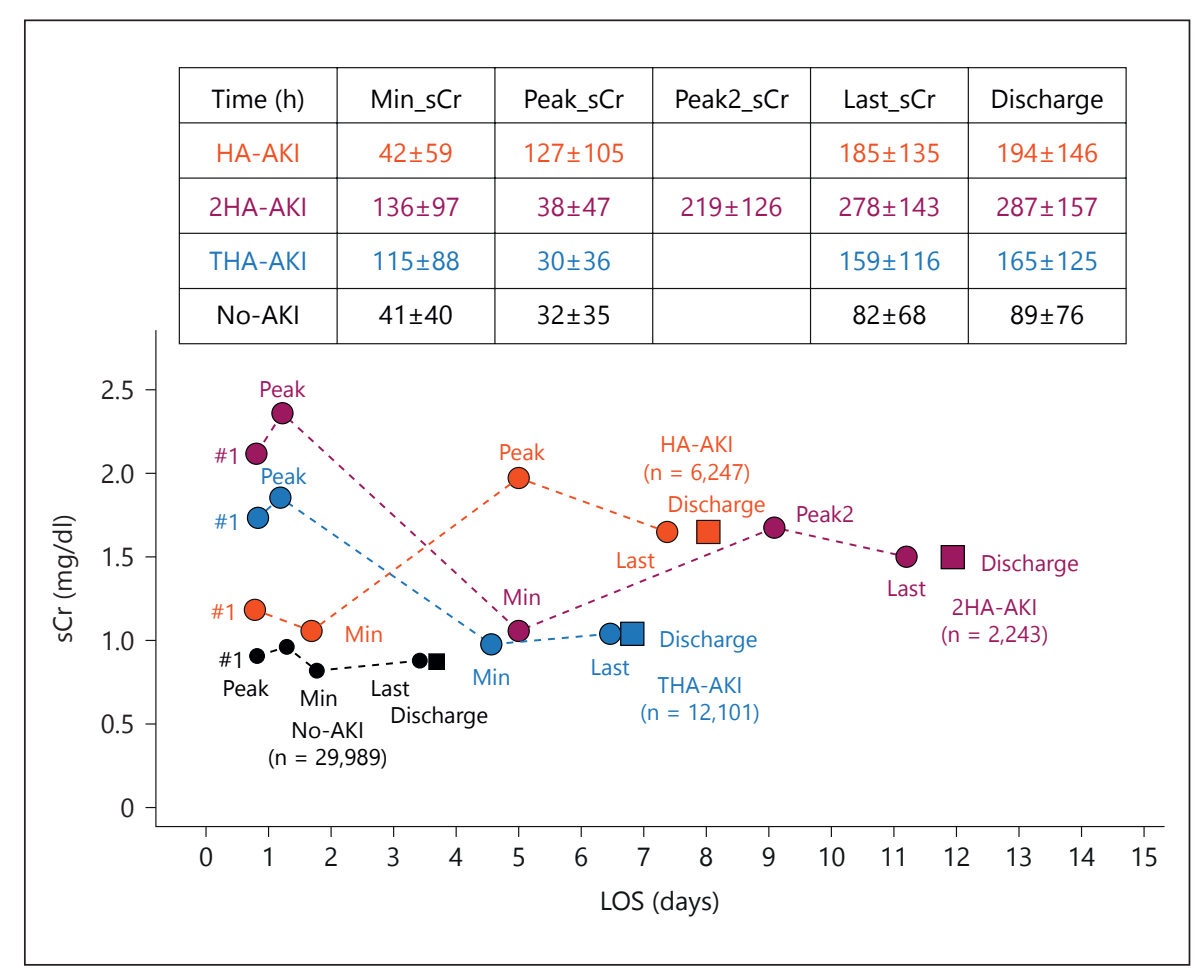

that occurred after they had recovered from the initial elevation of sCr present at admission (purple line; fig. 1). The peak $2 \mathrm{sCr}$ values needed for classification as $2 \mathrm{HA}$ AKI were defined as $\geq 0.3 \mathrm{mg} / \mathrm{dl}$ above the individual minimum sCr and occurred at $219 \pm 126 \mathrm{~h}(136 \pm 97 \mathrm{~h})$ for the 2HA-AKI patients. As seen in table 1, the baseline characteristics of these patients were more similar to the HA-AKI patients than to the THA-AKI patients, and they were more likely to receive inpatient RRT, had longer LOS and spent more days in an ICU than the other categories. Despite these differences, the 2HA-AKI patients had better short-term mortality outcomes than the HAAKI patients (table 2).

Table 2 summarizes recovery for patients with THAAKI, 2HA-AKI and HA-AKI. By definition, 100\% of the 2,243 2HA-AKI patients recovered from the initial peak $\mathrm{sCr}$ to the minimum $\mathrm{sCr}$, and thereafter developed a subsequent elevation of $\mathrm{sCr}$ that was $\geq 0.3 \mathrm{mg} / \mathrm{dl}$ above their individual minimum sCr value. Of the 12,101 THA-AKI patients, $80 \%$ had $>75 \%$ decrease from the peak sCr compared to their last sCr, and $<1 \%$ had $<33 \%$ improvement from their peak sCr value. In contrast, the improvement from the secondary peak sCr for 2HAAKI patients, and from peak sCr for HA-AKI patients was blunted, with $<20 \%$ of these patients showing $75 \%$ improvement from their peak sCr values. The majority of $2 \mathrm{HA}$-AKI (71\%) and HA-AKI (52\%) patients had less than modest improvement (>33\%) from their peak $\mathrm{sCr}$ values.

The relevant sCr values for each AKI category are summarized in table 2, as well as other baseline characteristics and inpatient mortality comparisons. For those patients who received care in an ICU, the average number of days in those units was $4.8 \pm 3.6$ days for patients with no AKI, compared to $>9$ days for patients with AKI. The inpatient mortality rates (No-AKI, 1.7\%; THA-AKI, 5\%; 2HAAKI, 12\%; HA-AKI, 18\%) increased in parallel with the number of ICU days, as well as LOS across the AKI categories. The last row in table 2 shows the HRs for the AKI categories, obtained with Cox multivariable regression analysis.

Table 3 summarizes the Cox proportional hazard models for inpatient mortality, with sequential addition of covariates and analysis of their time dependence, as reflected in the correlation (rho) of the smoothed Schoenfeld residuals with time and the significant interactions with $\ln (\mathrm{LOS})$. When the time-dependent covariates were include in the Cox model, the HR for THA-AKI was 1.088 (95\% CI 0.952-1.242; $\mathrm{p}=0.217$ ) and the HR for 2HA-AKI was 1.015 (95\% CI 0.84-1.223; $\mathrm{p}=0.868$ ). The HR for HA-AKI remained significantly different from zero and the other AKI HRs (table 3). Even though the time-aver- 
Table 2. Characteristics of 50,580 inpatients (2,500 deaths, $4.9 \%)$ without or with AKI, including sCr values (mg/dl)

\begin{tabular}{|c|c|c|c|c|}
\hline & \multicolumn{4}{|l|}{ AKI category } \\
\hline & $\begin{array}{l}\text { No-AKI: } \\
\text { 29,989 (59) }\end{array}$ & $\begin{array}{l}\text { THA-AKI: } \\
12,101(24)\end{array}$ & $\begin{array}{l}\text { 2HA-AKI: } \\
2,243 \text { (4) }\end{array}$ & $\begin{array}{l}\text { HA-AKI: } \\
6,247 \text { (12) }\end{array}$ \\
\hline LOS, days & $3.7 \pm 3.2$ & $6.9 \pm 5.2$ & $12 \pm 6.5$ & $8.1 \pm 6.1$ \\
\hline Age $>$ median ( 55 years) & $14,262(48)$ & $6,666(55)$ & $1,268(57)^{\mathrm{a}}$ & $3,798(61)$ \\
\hline HCF transfers & $6,336(21)$ & $3,237(25)$ & $426(33)$ & $2,005(32)^{\mathrm{a}}$ \\
\hline Minimum sCr & $0.8 \pm 0.3$ & $1.0 \pm 0.7$ & $1.1 \pm 1.0^{\mathrm{a}}$ & $1.1 \pm 0.7^{\mathrm{a}}$ \\
\hline Peak sCr & $1.0 \pm 0.3$ & $1.9 \pm 1.7$ & $2.3 \pm 2.6^{\mathrm{a}}$ & $2.0 \pm 1.5^{\mathrm{a}}$ \\
\hline 2nd peak sCr & & $0.9 \pm 0.4$ & $1.7 \pm 1.5$ & \\
\hline Last $\mathrm{sCr}$ & $0.9 \pm 0.3$ & $1.0 \pm 0.7$ & $1.5 \pm 1.4^{\mathrm{a}}$ & $1.6 \pm 0.3^{\mathrm{a}}$ \\
\hline sCr: number/day & $1.4 \pm 0.7$ & $1.5 \pm 0.8$ & $1.6 \pm 0.9$ & $1.4 \pm 0.9$ \\
\hline$<33 \%$ recovery 2 & $\mathrm{~N} / \mathrm{A}$ & & $1,594(71)$ & \\
\hline Charlson index & $1(0-2)$ & $1(0-2)$ & $1(0-2)$ & $2(0-2)$ \\
\hline Inpatient RRT & 0 & $287(2.4)$ & $246(11)$ & $256(4.1)$ \\
\hline Total ICU days & $4.8 \pm 3.6$ & $9.2 \pm 6.0$ & $13 \pm 6.6^{\mathrm{a}}$ & $9.8 \pm 6.6^{\mathrm{a}}$ \\
\hline Inpatient deaths & $506(1.7)$ & $606(5.0)$ & $267(12)$ & $1,121(18)^{\mathrm{a}}$ \\
\hline IR: death & $0.128(0.117-0.140)$ & $0.205(0.189-0.222)$ & $0.278(0.247-0.314)$ & $0.622(0.587-0.660)$ \\
\hline IRR: deaths & 1.00 (reference) & $1.60(1.42-1.80)$ & $2.18(1.88-2.52)$ & $4.86(4.38-5.40)^{\mathrm{a}}$ \\
\hline HR for death ${ }^{\mathrm{d}}$ & 1.00 (reference) & $1.23(1.09-1.40)$ & $1.27(1.07-1.51)^{\mathrm{a}}$ & $2.24(1.99-2.51)^{\mathrm{a}}$ \\
\hline
\end{tabular}

Unless otherwise indicated, values are given as number (percentage) or means $\pm 1 \mathrm{SD}$, medians are given with (25th-75thpercentile).

Baseline eGFR $<60$, baseline eGFR calculated from minimal sCr; risk category defined by patients with baseline eGFR $<60 \mathrm{ml} /$ $\mathrm{min} / 1.73 \mathrm{~m}^{2} ; \mathrm{IRR}=$ incident rate ratio (incident rates express as events per 28 patient-days).

$p<0.001$ for comparisons of characteristics between each group by ANOVA for means, signed-rank test for medians, and chi-square test for proportions; shown as bold text, relative to 'No-AKI', which served as the reference group.
${ }^{\mathrm{a}} \mathrm{p}<0.05$ for comparison between THA-AKI and HA-AKI (Dunnett test for multiple comparisons) or chi-square test of proportions.

${ }^{\mathrm{b}}$ eGFR calculated with minimum sCr.

c Percent recovery calculated as $100^{*}$ (peak sCr - minimum $\mathrm{sCr}) /($ peak sCr - last sCr).

${ }^{\mathrm{d}} \mathrm{HR}$ for inpatient mortality (Cox model; adjusted for median age, gender, race, Charlson comorbidity index, admission source, eGFR $<60$, percent recovery to $75 \%$, percent recover $<35 \%$, and RRT). aged HRs for THA-AKI and 2HA-AKI were not significantly from the reference category (No-AKI), there was a significant interaction between 2HA-AKI and elapsed time in the hospital.

Figure 2 shows the survival functions for the AKI categories with point-wise 95\% CI for the survival curves [17]. While the survival curve for HA-AKI clearly separated from the other categories early during the course of hospitalization, the survival curves for THA-AKI and 2HA-AKI only became evident for patients with prolonged LOSs. The curves for THA-AKI and 2HA-AKI were similar over time.

AKI and Inpatient Mortality

\section{Discussion}

We describe a retrospective analysis with 4 years ascertainment of all initial admissions to a single large academic referral medical center, and have used the timing of peak and minimal sCr values to distinguish between transient and more prolonged AKI during the hospital course. Over $40 \%$ of the admissions presented with AKI at the time of admission, or developed AKI after being admitted UAHB. The $24 \%$ of all qualifying admissions classified as TH-AKI (including the subset (4\%) of patients who presented with TH-AKI, but experienced re- 
Table 3. Cox multiple regression models: univariate analysis

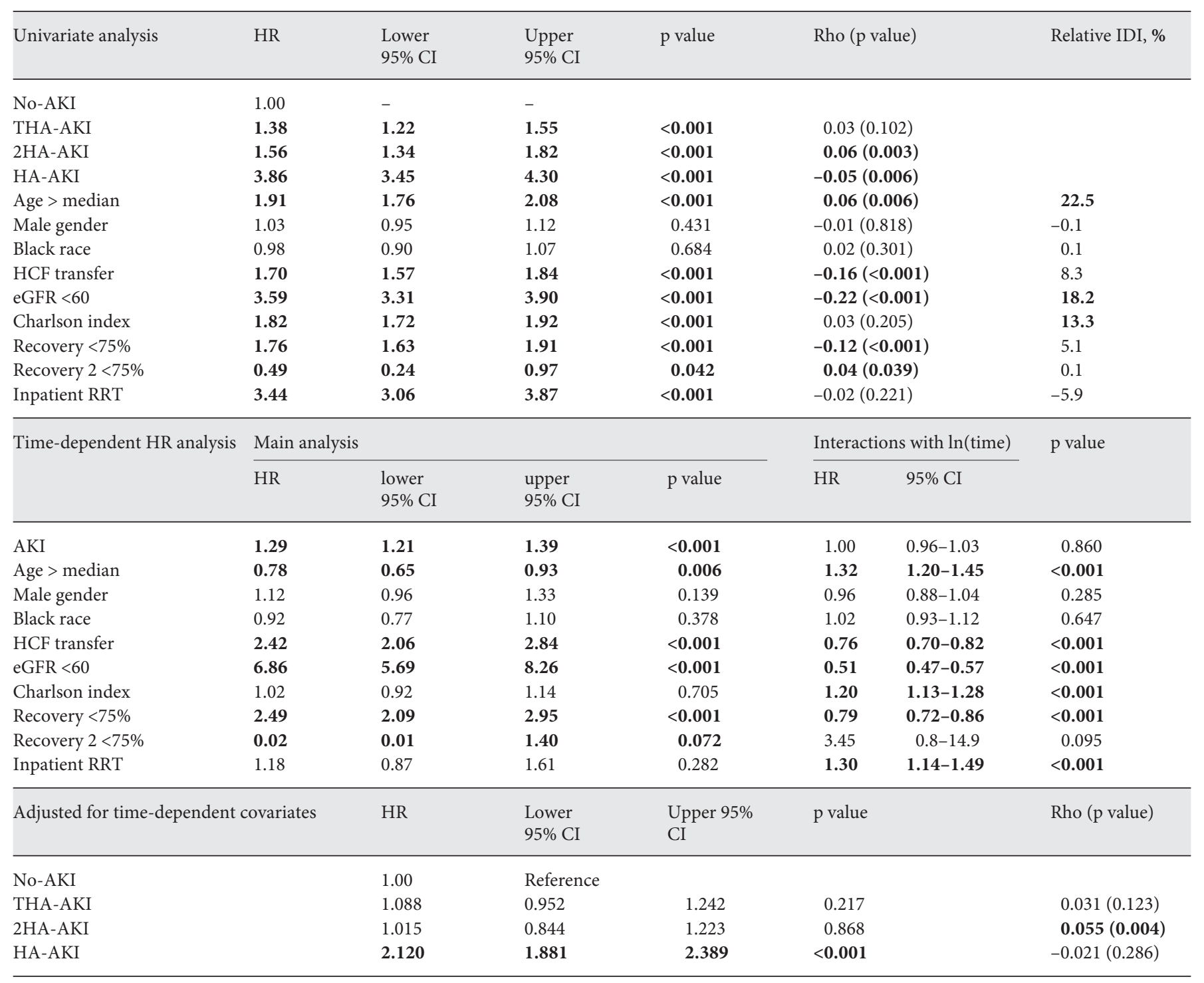

Age $>$ median, risk category for age $>55$ years (median value for entire cohort); eGFR $<60$, baseline eGFR calculated from minimal sCr, risk category defined by patients with baseline eGFR $<60 \mathrm{ml} / \mathrm{min} / 1.73 \mathrm{~m}^{2}$; HCF transfer, patient admitted to the University of Alabama Hospital to another HCF; IRR = incident rate ratio (incident rates express as events per 28 patient-days); Rho = correlation coefficient for smoothed Schoenfeld residuals with time.

$\mathrm{p}<0.001$ for comparisons of characteristics between each group by ANOVA for means, signed-rank test for medians, and chi-square test for proportions; shown as bold text, relative to 'No-AKI', which served as the reference group.

Percent recovery calculated as $100^{*}$ (peak $\mathrm{sCr}-$ minimum $\mathrm{sCr}$ )/(peak $\mathrm{sCr}-$ last $\mathrm{sCr}$ ).

Criteria for inclusion of covariates: age, race, gender and others with: univariate HR > 3.0; significant correlation coefficient (rho) for Schoenfeld residual $(\mathrm{p}<0.05)$; or rIDI $>10 \%$.

onset of HA-AKI) accounted for 70\% of all cases with AKI during the ascertainment interval.

Overall, our findings confirm the previous description of 'transient azotemia' [10]. We also describe a subset of patients with THA-AKI who have developed a second episode of AKI during the same admission, which we have termed '2HA-AKI'. This subgroup had increased inpatient mortality rates compared to THA-AKI patients who returned and remained at the minimum $\mathrm{sCr}$, but lower mortality rates that the larger group of patients with HAAKI. When adjusted for LOS and other covariates, the hazard rates for inpatient mortality were identical for the 


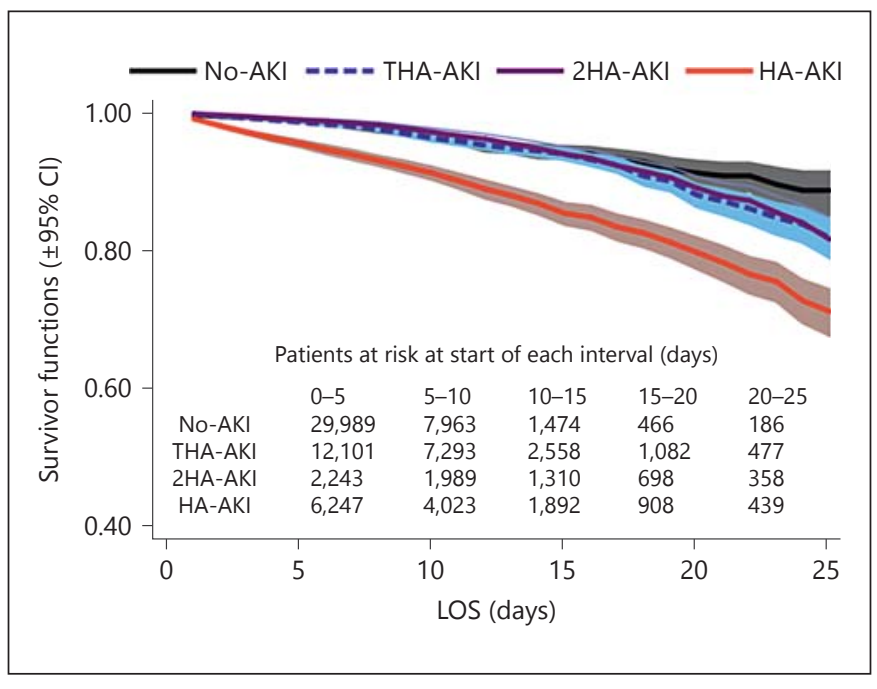

Fig. 2. Survival curves for AKI types. The analysis pertains to 50,601 patients with a single admission to the University of Alabama at Birmingham Hospital between October 1, 2009 and September 30, 2013. The survival curves were generated from Cox regression models, using point-wise confidence intervals for the covariate-adjusted survivor functions generated from Cox regression analysis [17]. \#1, first sCr recorded for admission; $2 \mathrm{HA}-\mathrm{AKI}$ defined by second peak $\mathrm{sCr} \geq 0.3 \mathrm{mg} / \mathrm{dl}$ above the minimum $\mathrm{sCr}$ with second peak sCr following the minimum sCr; HA-AKI defined as peak $\mathrm{s} C r \geq 0.3 \mathrm{mg} / \mathrm{dl}$ above the minimum $\mathrm{sCr}$ with minimum sCr preceding the peak sCr; last, last $\mathrm{sCr}$ recorded for admission; No-AKI defined as peak $\mathrm{sCr}<0.3 \mathrm{mg} / \mathrm{dl}$ above the minimum sCr; THA-AKI defined by peak $\mathrm{sCr} \geq 0.3 \mathrm{mg} / \mathrm{dl}$ above the minimum sCr with peak sCr preceding the minimum sCr. The patients at risk for each category relative to admission date are listed in the inset.

THA-AKI and 2HA-AKI groups, and significantly less than the HA-AKI group. The mortality risk for the 2HAAKI group may reflect the case-mix, comorbidities and other baseline characteristics and needs to be assessed in other independent data sets.

The subgroup with 2HA-AKI was not addressed in the previous description of transient azotemia, although a group with THA-AKI who had not improved by the third hospital day was noted and reclassified as 'persisting ATN' [10]. We suspect that this group with poor recovery from THA-AKI may well have been the group we now characterized as $2 \mathrm{HA}$-AKI. In future studies, it may be informative to evaluate these classifications of inpatient $\mathrm{AKI}$ and the risks for developing $\mathrm{AKI}$ as well as all-cause mortality during subsequent hospitalizations [20].

There are different assumptions that may account for the difference in apparent incidence of AKI and the ex- tent of improvement from peak sCr. Uchino et al. [10] defined transient azotemia as resolving within 3 days without RRT, while patients receiving RRT were classified as persisting ATN. We have not used extent of recovery in the present definitions of THA-AKI, 2HA-AKI and HA-AKI, and observed that $17 \%$ of patients with HAAKI had significant improvement $(\geq 80 \%)$ from their peak sCr values before hospital discharge and thus did not have persisting AKI. Sensitivity analysis showed that exclusion of patients receiving RRT did not change the estimates of recovery from AKI (data not shown); rather than reclassifying these patients as HA-AKI, we simply included RRT as a covariate in the time-to-event regression model. The major reason for the apparent increased incidence of AKI in our series was the use of a lower threshold $(0.3 \mathrm{mg} / \mathrm{dl})$ for diagnosing AKI $[9,14]$, rather than the higher $0.5 \mathrm{mg} / \mathrm{dl}$ used by Uchino et al. [10]. Neither study used prior measurements of $\mathrm{sCr}$ as baseline values; we used the minimal $\mathrm{sCr}$ for each admission, and Uchino et al. [10] imputed the baseline $\mathrm{sCr}$ based on an estimated eGFR of $75 \mathrm{ml} / \mathrm{min} / 1.73 \mathrm{~m}^{2}$ for two-thirds of their patients, an approach likely to overestimate that incidence of AKI [7, 8], and thus does not account for the increased incidence of AKI that we describe in the present analysis.

The definition of baseline kidney function is somewhat controversial even with the availability of previous ambulatory determinations of $\mathrm{sCr}[9,21,22]$. Using a preadmission ambulatory sCr as the baseline has been advocated, with a number of surrogates considered when ambulatory data is not available [21]. Siew et al. [7,8] defined the most recent ambulatory $\mathrm{sCr}$ measurement as the baseline sCr and found $\mathrm{AKI}$ incidence rates were underestimated for adults when the first admission $\mathrm{sCr}$ was used for the baseline (14\%), compared to the AKI incidence rate of $26 \%$ based on the last pre-admission sCr. The minimum $\mathrm{sCr}$ during admission performed better as a surrogate baseline $\mathrm{sCr}$ than the first measured $\mathrm{sCr}$, which may reflect a number of cases with THA-AKI included in their analyses. The misclassification and overestimation of the incidence of AKI that occurred when the minimum $\mathrm{sCr}$ was used as the surrogate baseline $\mathrm{sCr}$ was most evident in the mildest stages of AKI (i.e., stage 1).

There are 2 limitations of the current definitions of baseline sCr that are worth considering [23]: (1) AKI is a global health issue $[24,25]$, and the majority of patients with $\mathrm{AKI}$ in developing countries have never had a previous sCr measurement and (2) pre-hospitalization sCr values, when available, are only relevant to the magnitude of 
the baseline sCr, but do not address the timing of the baseline $\mathrm{sCr}$, which is the distinctive feature of the current analysis. Not only was the elapsed time from admission to the minimum sCr measurement significantly different, but there was also a statistically significant difference in the magnitude of the minimum sCr for patients with THA-AKI compared to HA-AKI. This observation questions whether there is a single baseline creatinine that is appropriate for all forms of AKI.

Finally, we report that there is only a modest risk for inpatient mortality associated with THA-AKI and 2HAAKI (fig. 2). If the regression analyses used time-averaged covariates, then the significance of the associate of inpatient mortality with THA-AKI and 2HA-AKI was greatly attenuated (table 3 ). The time dependence of hazards associated with AKI has been described in several recent publications [26-28]. Even though the HR for inpatient mortality was identical for THA-AKI and 2HA-AKI, the long-term outcomes may diverge, especially noting that the THA-AKI had nearly complete recovery from AKI, while the $2 \mathrm{HA}$-AKI group had severely reduced recovery from AKI, similar to the HA-AKI group.

Other reports have described AKI acquired before admission, which resolved in the hospital setting as 'community-acquired' AKI [22, 29-33]. We have operationally defined THA-AKI as cases where the peak sCr values precede the subsequent minimal $\mathrm{sCr}$ for that admission, and surmise that the rapid decrease in $\mathrm{sCr}$ may have reflected dehydration prior to admission that was promptly and appropriately corrected with fluid administration. Of the 14,344 patients with THA-AKI and 2HA-AKI in our analysis, 10,681 (75\%) were admitted to the UAHB from a community-based setting, but $25 \%$ were transferred/ admitted to the UAHB from other hospitals and HCFs; so THA-AKI and community-acquired AKI are not entirely synonymous. In contrast, a higher percentage of HA-AKI patients (32\%) were transferred to the UAHB from other HCFs.

Others have described inpatient recovery of AKI but the criteria for recovery have not been standardized [34]. Recovery to the baseline sCr measured before AKI developed has been used [5], often with the focus on severely ill patients in the critical care setting [35]. We report that the average improvement in elevated $\mathrm{sCr}$ for patients with THA-AKI was $88 \pm 18 \%$, with $80 \%$ achieving $75 \%$ improvement from the peak sCr before hospital discharge. In contrast, only $4.8 \%$ of the $2 \mathrm{HA}-\mathrm{AKI}$ and $17 \%$ of the HA-AKI achieved a 75\% reduction from the peak sCr before hospital discharge.
Our study has several limitations and is based on retrospective analyses of an administrative data set reflecting the 4-year experience at a single tertiary academic referral medical center. Information about pre-hospitalization $\mathrm{sCr}$ was not available, and neither can the etiology of AKI nor urine output nor fluid administration be determined from the administrative data set. Follow-up status after discharge was not included, and many patients, especially those with HA-AKI and 2HA-AKI, were discharged before they may have had more complete recovery of their renal function. We cannot describe their subsequent course due to the limitations of our data set. The density and timing of inpatient $\mathrm{sCr}$ data reflect case ascertainment that is dependent on clinical decision-making and laboratory testing ordering practices [3], which introduces several sources of potential confounding and bias [36].

Changes in $\mathrm{sCr}$ depend on factors that modify creatinine generation (body composition, muscle wasting and acute muscle injury) [37] and changes in the volume of distribution of creatinine (dehydration or volume expansion) may occur, especially in elderly [11] and critically ill patients with prolonged hospitalizations [38, 39]. Most admissions were not associated with significant changes $(\leq 0.3 \mathrm{mg} / \mathrm{dl})$ in $\mathrm{sCr}$; these admissions were defined as the reference group with 'No-AKI' for these analyses. We do not attribute the observed decreases in $\mathrm{sCr}$ to changes in creatinine generation rate because we censored each case at 28 days LOS, and the majority of the cases of THA-AKI were not exposed to an intensive care setting. Nevertheless, there is certainly a subset of critically ill patients who may have decreased creatinine generation rates, and net cumulative fluid balance, which could dilute their sCr values [40].

The approach described herein is readily applicable to retrospective analyses of large administrative inpatient data sets, and does not use diagnosis-related groups to define AKI categories. Further work is be needed to directly compare the minimum inpatient $\mathrm{s} C \mathrm{Cr}$ with previous values, to determine if the minimum inpatient $\mathrm{sCr}$ value can be used as a real-time tool in assessing the development and recovery from AKI during an acute hospitalization and to compare the minimum inpatient $\mathrm{s} C \mathrm{Cr}$ value to sCr values obtained for patients with previous hospital admissions.

In conclusion, we conducted a retrospective analysis of a large administrative set from a single academic medical center, and described 3 unique subgroups of AKI that were differentiated by the time course of the changes in sCr with reference to the minimal and maximal values 
obtained during a single hospitalization. While the associate risk for inpatient mortality was attenuated for THAAKI and 2HA-AKI, the finding that approximately $20 \%$ of patients initially classified as THA-AKI experienced a second episode of AKI suggests that continued surveillance of inpatient $\mathrm{s} C \mathrm{r}$ even after 'complete' recovery from THA-AKI would be informative and could potentially lead to clinical initiatives targeted at improving outcomes for this high risk group of patients. Finally, approximately $20 \%$ of patients with HA-AKI have significant improvement from their peak $\mathrm{sCr}$ values during the hospitalization. More information is needed to better characterize recovery from peak $\mathrm{sCr}$ in patients with $\mathrm{AKI}$, with the goals of better surveillance and implementation of pro-active treatment programs that could positively impact patient outcomes and LOS [23].

\section{Acknowledgments}

This paper was presented at the AKI as a Quality Paradigm: Round Table Conference at the 20 International Conference on Continuous Renal Replacement Therapies (Manchester Grand Hyatt, San Diego, Calif., USA, February 15-16, 2015).

We thank Darlene Green and Stephen Duncan for their assistance with the data set, and assistance and provision of the Stata macro for relative IDI [41] from Kunihiro Matsushita and Ying
Yang Sang of the CKD-Prognosis Consortium (http://www.jhsph. edu/research/centers-and-institutes/chronic-kidney-diseaseprognosis-consortium/index.html).

\section{Authorship}

All authors participated in the study design, analysis and writing of the manuscript. No other persons participated in the analyses, drafting or revision of the manuscript.

\section{Support}

A Kidney Research Student Scholar Grant from the American Society of Nephrology Foundation supported Mr. T.C. Powell. J.P. Donnelly received support from the Agency for Healthcare Research and Quality, Rockville, Maryland (T32-HS013852). Dr. H.E. Wang received grant support from the National Institute for Nursing Research (R01-NR012726). The Hilda B. Anderson Endowed Professorship in Nephrology supported Dr. D.G. Warnock. We also acknowledge support from the UAB/UCSD O'Brien Center Kidney Research (P30 DK079337).

\section{Disclosure Statement}

None of the authors disclose any conflicts of interest relevant to this report.

\section{References}

1 Chawla LS, Eggers PW, Star RA, Kimmel PL: Acute kidney injury and chronic kidney disease as interconnected syndromes. N Engl J Med 2014;371:58-66.

-2 Bagshaw SM, Laupland KB, Doig CJ, Mortis G, Fick GH, Mucenski M, Godinez-Luna T, Svenson LW, Rosenal T: Prognosis for longterm survival and renal recovery in critically ill patients with severe acute renal failure: a population-based study. Crit Care 2005;9: R700-R709.

-3 Chertow GM, Burdick E, Honour M, Bonventre JV, Bates DW: Acute kidney injury, mortality, length of stay, and costs in hospitalized patients. J Am Soc Nephrol 2005;16:3365-3370.

4 Wang HE, Muntner P, Chertow GM, Warnock DG: Acute kidney injury and mortality in hospitalized patients. Am J Nephrol 2012; 35:349-355.

5 Meersch M, Schmidt C, Van Aken H, Martens S, Rossaint J, Singbartl K, Gorlich D, Kellum JA, Zarbock A: Urinary TIMP-2 and IGFBP7 as early biomarkers of acute kidney injury and renal recovery following cardiac surgery. PLoS One 2014;9:e93460.

6 Hsu CY, McCulloch CE, Fan D, Ordonez JD, Chertow GM, Go AS: Community-based in- cidence of acute renal failure. Kidney Int 2007;72:208-212.

$\checkmark 7$ Siew ED, Matheny ME: Choice of reference serum creatinine in defining acute kidney injury. Nephron 2015;131:107-112.

8 Siew ED, Matheny ME, Ikizler TA, Lewis JB, Miller RA, Waitman LR, Go AS, Parikh CR, Peterson JF: Commonly used surrogates for baseline renal function affect the classification and prognosis of acute kidney injury. Kidney Int 2010;77:536-542.

9 KDIGO clinical practice guideline for acute kidney injury. Kidney Int Suppl 2012;2:1138.

10 Uchino S, Bellomo R, Bagshaw SM, Goldsmith D: Transient azotaemia is associated with a high risk of death in hospitalized patients. Nephrol Dial Transplant 2010;25: 1833-1839.

11 Chao CT, Wu VC, Lai CF, Shiao CC, Huang TM, Wu PC, Tsai IJ, Hou CC, Wang WJ, Tsai HB, Lin YF, Chiang WC, Lin SL, Tsai PR, Ko WJ, Wu MS, Wu KD; NSARF group: Advanced age affects the outcome-predictive power of RIFLE classification in geriatric patients with acute kidney injury. Kidney Int 2012;82:920-927.
2 Fidalgo P, Ahmed M, Meyer SR, Lien D, Weinkauf J, Kapasi A, Cardoso FS, Jackson K, Bagshaw SM: Association between transient acute kidney injury and morbidity and mortality after lung transplantation: a retrospective cohort study. J Crit Care 2014;29:1028-1034.

13 Point of Origin for Admission or Visit Codes Update to the UB-04 (CMS-1450); in Center for Medicare and Medicaid Services, 2010. https://www.cms.gov/Outreach-andEducation/Medicare-Learning-NetworkMLN/MLNMattersArticles/downloads/ MM6801.pdf (accessed July 10, 2015).

14 Broce JC, Price LL, Liangos O, Uhlig K, Jaber BL: Hospital-acquired acute kidney injury: an analysis of nadir-to-peak serum creatinine increments stratified by baseline estimated GFR. Clin J Am Soc Nephrol 2011;6:15561565.

15 Deyo RA, Cherkin DC, Ciol MA: Adapting a clinical comorbidity index for use with ICD9-CM administrative databases. J Clin Epidemiol 1992;45:613-619.

16 Stevens LA, Coresh J, Greene T, Levey AS: Assessing kidney function - measured and estimated glomerular filtration rate. $\mathrm{N}$ Engl J Med 2006;354:2473-2483. 
17 Cefalu MS: Pointwise confidence intervals for the covariate-adjusted survivor function in the Cox model. Stata J 2011;11:64-81.

- 18 Pencina MJ, D’Agostino RB Sr, D’Agostino RB Jr, Vasan RS: Evaluating the added predictive ability of a new marker: from area under the ROC curve to reclassification and beyond. Stat Med 2008;27:157-172; discussion 207212.

19 Pencina MJ, D’Agostino RB Sr, Steyerberg EW: Extensions of net reclassification improvement calculations to measure usefulness of new biomarkers. Stat Med 2011;30:11-21.

20 Siew ED, Parr SK, Abdel-Kader K, Eden SK, Peterson JF, Bansal N, Hung AM, Fly J, Speroff T, Ikizler TA, Matheny ME: Predictors of recurrent AKI. J Am Soc Nephrol 2015;pii: ASN.2014121218.

21 Ricci Z, Cruz DN, Ronco C: Classification and staging of acute kidney injury: beyond the RIFLE and AKIN criteria. Nat Rev Nephrol 2011;7:201-208.

22 Wonnacott A, Meran S, Amphlett B, Talabani B, Phillips A: Epidemiology and outcomes in community-acquired versus hospital-acquired AKI. Clin J Am Soc Nephrol 2014;9: 1007-1014.

23 Powell TC, Warnock DG: The furosemide stress test and predicting AKI outcomes. J Am Soc Nephrol 2015;26:1762-1764.

-24 Susantitaphong P, Cruz DN, Cerda J, Abulfaraj M, Alqahtani F, Koulouridis I, Jaber BL; Acute Kidney Injury Advisory Group of the American Society of Nephrology: World incidence of AKI: a meta-analysis. Clin J Am Soc Nephrol 2013;8:1482-1493.

25 Horton R, Berman P: Eliminating acute kidney injury by 2025: an achievable goal. Lancet 2015;385:2551-2552.

$26 \mathrm{Wu} \mathrm{VC}, \mathrm{Wu} \mathrm{CH}$, Huang TM, Wang CY, Lai CF, Shiao CC, Chang CH, Lin SL, Chen YY,
Chen YM, Chu TS, Chiang WC, Wu KD, Tsai PR, Chen L, Ko WJ; NSARF Group: Longterm risk of coronary events after AKI. J Am Soc Nephrol 2014;25:595-605.

27 Wu VC, Wu PC, Wu CH, Huang TM, Chang $\mathrm{CH}$, Tsai PR, Ko WJ, Chen L, Wang CY, Chu $\mathrm{TS}, \mathrm{Wu} \mathrm{KD}$; National Taiwan University Study Group on Acute Renal Failure (NSARF) Group: The impact of acute kidney injury on the long-term risk of stroke. J Am Heart Assoc 2014;pii:e000933.

28 Suzuki S, Eastwood GM, Bailey M, Gattas D, Kruger P, Saxena M, Santamaria JD, Bellomo $\mathrm{R}$ : Paracetamol therapy and outcome of critically ill patients: a multicenter retrospective observational study. Crit Care 2015;19:162.

29 Srisawat N, Murugan R, Lee M, Kong L, Carter M, Angus DC, Kellum JA; Genetic and Inflammatory Markers of Sepsis (GenIMS) Study Investigators: Plasma neutrophil gelatinase-associated lipocalin predicts recovery from acute kidney injury following community-acquired pneumonia. Kidney Int 2011 80:545-552.

30 Murugan R, Weissfeld L, Yende S, Singbartl K, Angus DC, Kellum JA; Genetic and Inflammatory Markers of Sepsis (GenIMS) Investigators: Association of statin use with risk and outcome of acute kidney injury in community-acquired pneumonia. Clin J Am Soc Nephrol 2012;7:895-905.

31 Schissler MM, Zaidi S, Kumar H, Deo D, Brier ME, McLeish KR: Characteristics and outcomes in community-acquired versus hospital-acquired acute kidney injury. Nephrology (Carlton) 2013;18:183-187.

32 Der Mesropian PJ, Kalamaras JS, Eisele G, Phelps KR, Asif A, Mathew RO: Long-term outcomes of community-acquired versus hospital-acquired acute kidney injury: a retrospective analysis. Clin Nephrol 2014;81:174-184.
33 Barton AL, Mallard AS, Parry RG: One year's observational study of acute kidney injury incidence in primary care: frequency of followup serum creatinine and mortality risk. Nephron 2015;130:175-181.

34 Macedo E, Bouchard J, Mehta RL: Renal recovery following acute kidney injury. Curr Opin Crit Care 2008;14:660-665.

35 Coca SG: Is it AKI or nonrecovery of renal function that is important for long-term outcomes? Clin J Am Soc Nephrol 2013;8:173176.

36 Coca SG, Peixoto AJ, Garg AX, Krumholz HM, Parikh CR: The prognostic importance of a small acute decrement in kidney function in hospitalized patients: a systematic review and meta-analysis. Am J Kidney Dis 2007;50: 712-720.

-37 Star RA: Treatment of acute renal failure. Kidney Int 1998;54:1817-1831.

38 Doi K, Yuen PS, Eisner C, Hu X, Leelahavanichkul A, Schnermann J, Star RA: Reduced production of creatinine limits its use as marker of kidney injury in sepsis. J Am Soc Nephrol 2009;20:1217-1221.

39 Prowle JR, Kolic I, Purdell-Lewis J, Taylor R, Pearse RM, Kirwan CJ: Serum creatinine changes associated with critical illness and detection of persistent renal dysfunction after AKI. Clin J Am Soc Nephrol 2014;9:10151023.

40 Macedo E, Bouchard J, Soroko SH, Chertow GM, Himmelfarb J, Ikizler TA, Paganini EP, Mehta RL; Program to Improve Care in Acute Renal Disease Study: Fluid accumulation, recognition and staging of acute kidney injury in critically-ill patients. Crit Care 2010;14:R82.

41 Chambless LE, Cummiskey CP, Cui G: Several methods to assess improvement in risk prediction models: extension to survival analysis. Stat Med 2011;30:22-38. 